\title{
A D-Band Rectangular Waveguide-to-Coplanar Waveguide Transition Using Wire Bonding Probe
}

Dong, Yunfeng; Zhurbenko, Vitaliy; Hanberg, Peter Jesper; Johansen, Tom Keinicke

Published in:

Journal of Infrared, Millimeter and Terahertz Waves

Link to article, DOI:

$10.1007 / \mathrm{s} 10762-018-0551-x$

Publication date:

2018

Document Version

Peer reviewed version

Link back to DTU Orbit

Citation (APA):

Dong, Y., Zhurbenko, V., Hanberg, P. J., \& Johansen, T. K. (2018). A D-Band Rectangular Waveguide-toCoplanar Waveguide Transition Using Wire Bonding Probe. Journal of Infrared, Millimeter and Terahertz Waves. https://doi.org/10.1007/s10762-018-0551-x

\section{General rights}

Copyright and moral rights for the publications made accessible in the public portal are retained by the authors and/or other copyright owners and it is a condition of accessing publications that users recognise and abide by the legal requirements associated with these rights.

- Users may download and print one copy of any publication from the public portal for the purpose of private study or research.

- You may not further distribute the material or use it for any profit-making activity or commercial gain

- You may freely distribute the URL identifying the publication in the public portal 


\title{
A D-Band Rectangular Waveguide-to-Coplanar Waveguide Transition Using Wire Bonding Probe
}

\author{
Yunfeng Dong • Vitaliy Zhurbenko • \\ Peter J. Hanberg • Tom K. Johansen
}

Received: date / Accepted: date

\begin{abstract}
This paper presents a rectangular waveguide-to-coplanar waveguide $(\mathrm{CPW})$ transition at D-band (110-170 GHz) using wire bonding probe. A conventional CPW is designed and fabricated based on both quartz and glass substrates for estimating the losses at D-band and testing the fabrication processes. Two transition prototypes at D-band using E-plane probe and wire bonding probe are designed, fabricated, and measured in a back-to-back configuration. The system packaging approaches and scattering parameters of the transition prototypes are compared. For both on-chip and carrier substrate approaches, chips can be tested individually before packaged into a system and extra connections are not required. The fabricated rectangular waveguide-toCPW transition at D-band using wire bonding probe in a back-to-back configuration exhibits a bandwidth of $56.3 \mathrm{GHz}$ ranging from $110 \mathrm{GHz}$ to $166.3 \mathrm{GHz}$ in which the return loss is better than $10 \mathrm{~dB}$ with an associated insertion loss of $2 \mathrm{~dB}$. Compared with the fabricated rectangular waveguide-to-CPW transition using E-plane probe, an equivalent bandwidth is achieved at D-band while the system packaging approaches are more versatile and compact for the proposed transition using wire bonding probe.
\end{abstract}

Keywords Coplanar waveguide (CPW) · E-plane probe · Packaging · Rectangular waveguide $\cdot$ Wideband transition - Wire bonding probe

Yunfeng Dong

E-mail: yundon@elektro.dtu.dk

Yunfeng Dong, Vitaliy Zhurbenko, Tom K. Johansen

Electromagnetic Systems Group, Department of Electrical Engineering, Technical University of Denmark, Oersteds Plads Building 348, DK-2800 Kgs. Lyngby, Denmark

Peter J. Hanberg

National Center for Micro- and Nanofabrication, Technical University of Denmark, Oersteds Plads Building 347, DK-2800 Kgs. Lyngby, Denmark 


\section{Introduction}

Due to low loss and high power-handling capability, waveguides are widely used in millimeter-wave and submillimeter-wave systems. The width and height of rectangular waveguides are defined by frequency bands and it is used as the standardized interface for cascading different components and systems. For applications at high frequencies, rectangular waveguides become small in size and require accurate fabrication. In addition to milling, other technologies such as low-temperature co-fired ceramic (LTCC), 3D printing, and PolyStrata are also used for fabrications [1]-[3].

Though rectangular waveguides are used for packaging millimeter-wave and submillimeter-wave circuits, the components and chips at such high frequencies are mainly relied on planar structures due to their fabrication processes. Coplanar waveguide (CPW) and microstrip are two types of planar transmission lines commonly used for designing high frequency chips. Due to the need for system packaging, the transitions between rectangular waveguides and planar transmission lines are under intensive study [4]-[20]. The challenging requirements for such transitions are wideband operation with low insertion loss and at the same time being versatile and compact for packaging the existing components and chips. In [4]-[6], rectangular waveguide-to-CPW transitions were realized by tapering the height and width of the waveguide in several stages to match the characteristic impedance of the CPW. With micromachining technologies, a short-circuited probe can be fabricated connecting the conductor wall of the tapered rectangular waveguide to the CPW. As is reported in [7]-[9], by inserting antennas at the end of the rectangular waveguide in the direction of maximum radiation the electromagnetic waves are guided from rectangular waveguide to CPW and microstrip. The antenna is patterned on the substrate and the bandwidth of the transition is limited by the designed wideband antenna. Among other types of transitions, E-plane probe exhibit noticeable performances for the circuits based on CPW and microstrip especially at millimeter-wave and submillimeter-wave frequencies [10]-[19]. The probe is patterned on a substrate inserting into a rectangular waveguide through an aperture cut in the center of the broadwall parallel to the longitudinal axis. Its location is about quarter wavelength away from the end of the rectangular waveguide forming an impedance transformer converting the termination from short to open. Since the inserted probe is in parallel with the electric fields inside the rectangular waveguide, it is called E-plane probe transition. According to the published results in the literature, the E-plane probes can be designed with different shapes and the probe with a rectangular patch is found to be very effective which by carefully designing can achieve a smooth transmission within a whole waveguide frequency band. Though E-plane probe transitions can provide wideband operations with low insertion loss, it still faces difficulties for system packaging. When E-plane probes are designed on-chip, the chips cannot be tested or adjusted individually before packaged into systems. For packaging the existing components and chips, extra connections are required between E-plane probes and chips which 


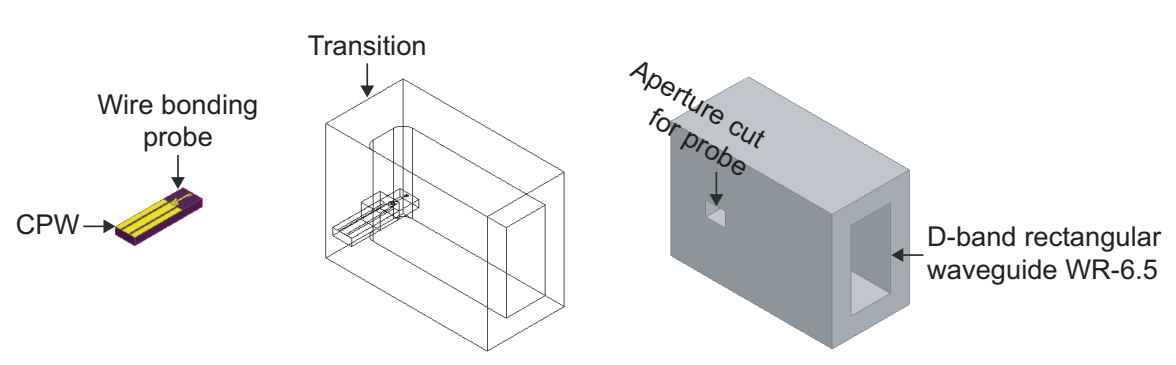

Fig. 1 Assembly structure of the proposed transition between a WR-6.5 rectangular waveguide and a CPW using wire bonding as a probe.

limit the bandwidth of the transitions as well as systems.

In this paper, a D-band rectangular waveguide-to-CPW transition using wire bonding probe is proposed. Fig. 1 shows the assembly structure of the proposed transition between a WR-6.5 rectangular waveguide and a CPW using wire bonding as a probe. The CPW in the assembly structure represents the interface of millimeter-wave and submillimeter-wave circuits. Except for CPW, the proposed wire bonding probe is also compatible with other types of planar transmission lines such as microstrip. The organization of the paper is as follows. In Sect. 2, the designs of a conventional CPW as well as the CPW packaged inside an aluminum cover are introduced. The measurement results of the designed CPW based on both quartz and glass substrates are shown and compared. In Sect. 3, the proposed rectangular waveguide-to-CPW transitions at D-band using E-plane probe and wire bonding probe are described. Besides, the system packaging approaches by using the proposed transitions are discussed. In Sect. 4, the fabrication and experimental results of the downscaled transitions at U-band and the proposed transitions at D-band are presented. Conclusions are finally drawn in Sect. 5 .

\section{CPW Design Considerations}

\subsection{Selecting CPW Parameters and Substrate Material}

When designing a planar transmission line at high frequencies, not only the characteristic impedance but also the attenuation due to using non-ideal materials need to be taken into consideration. The conductor loss is caused by the current flow inside the transmission line conductor. The thickness of the conductor is a compromise between the skin depth and the expense since the circuits at high frequencies normally use silver, gold, or titanium as conductors. The dielectric loss is caused by the substrate material and it is determined by the dissipation factor $(\tan \delta)$ which varies based on the operating frequency. There is a risk when using a substrate directly for designing circuits at high frequencies since the $\tan \delta$ of the substrate is normally measured at low frequencies by the supplier. In order to estimate the losses caused by the conductor as well as the substrate materials at D-band and to test the fabrication processes, the 


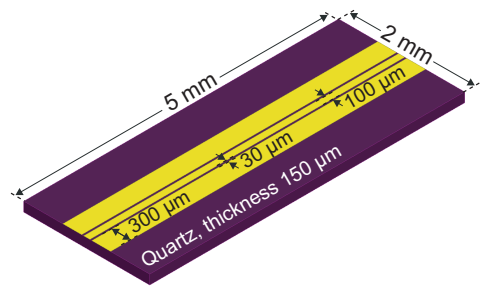

(a)
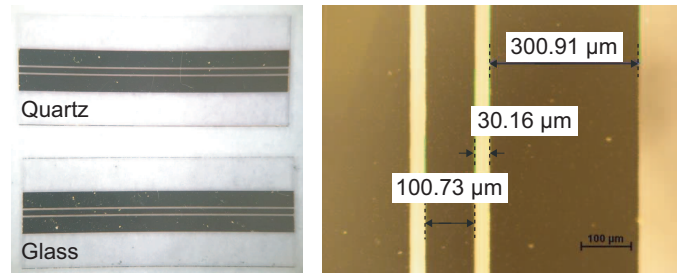

(b)

Fig. 2 a Designed conventional CPW. b Fabricated CPWs based on quartz and glass substrates.

conventional CPW shown in Fig. 2a is designed and fabricated based on both quartz and glass substrates. The conventional $\mathrm{CPW}$ consists of two ground traces and a signal trace forming a ground-signal-ground structure. A quasiTEM mode can be supported and the electric fields mainly exist in the gaps between the signal and ground traces. Unlike microstrip whose characteristic impedance is determined by the substrate and the width of the transmission line, the characteristic impedance of a conventional CPW is mainly related to the ratio of $S /(S+2 G)$ in which $S$ and $G$ represent the width of the signal trace and the width of the gap, respectively. Though the width of the ground trace does not affect the characteristic impedance directly, it needs to be wide enough for supporting the propagation of electromagnetic waves and at the same time it should not cause resonances within the designed frequency band especially for conductor-backed CPWs [21]-[23].

For the designed conventional CPW, the width of the signal trace is $100 \mu \mathrm{m}$ and the width of the ground trace is $300 \mu \mathrm{m}$ with a gap width of $30 \mu \mathrm{m}$. The material of the conductor layer is gold and its thickness is $400 \mathrm{~nm}$. Though the major ingredient of both quartz and glass substrates is silicon dioxide $\left(\mathrm{SiO}_{2}\right)$, they have different percentages of impurities which affects the $\tan \delta$ especially at high frequencies. The thickness of the substrates is $150 \mu \mathrm{m}$ and the dielectric constant $\left(\epsilon_{r}\right)$ is 4 . The conductor layer was deposited using gold sputtering at Danchip (National Center for Micro- and Nanofabrication in Denmark). In order to improve the adhesion of the gold conductor, a titanium layer with a thickness of $30 \mathrm{~nm}$ was deposited first on top of the substrates. The CPW was patterned by laser ablation using a picosecond laser with a wavelength of $355 \mathrm{~nm}$ focused down to a spot size of approximate $10 \mu \mathrm{m}$. After that, the substrates were diced into pieces with a length of $5 \mathrm{~mm}$ and a width of $2 \mathrm{~mm}$. Fig. $2 \mathrm{~b}$ shows the fabricated CPWs and the measured dimensions by using a microscope.

The blue and red lines in Fig. 3 show the measurement results of the fabricated CPWs based on quartz and glass substrates, respectively. An insertion loss of $0.254 \mathrm{~dB} / \mathrm{mm}$ has been achieved by the CPW based on quartz substrate at $140 \mathrm{GHz}$ while it is $1 \mathrm{~dB} / \mathrm{mm}$ for the $\mathrm{CPW}$ based on glass substrate. Since the substrate material is the only difference between the two fabricated CPWs, the quartz substrate has proved its low-loss transmission at D-band, thus it is used for designing rectangular waveguide-to-CPW transitions. 


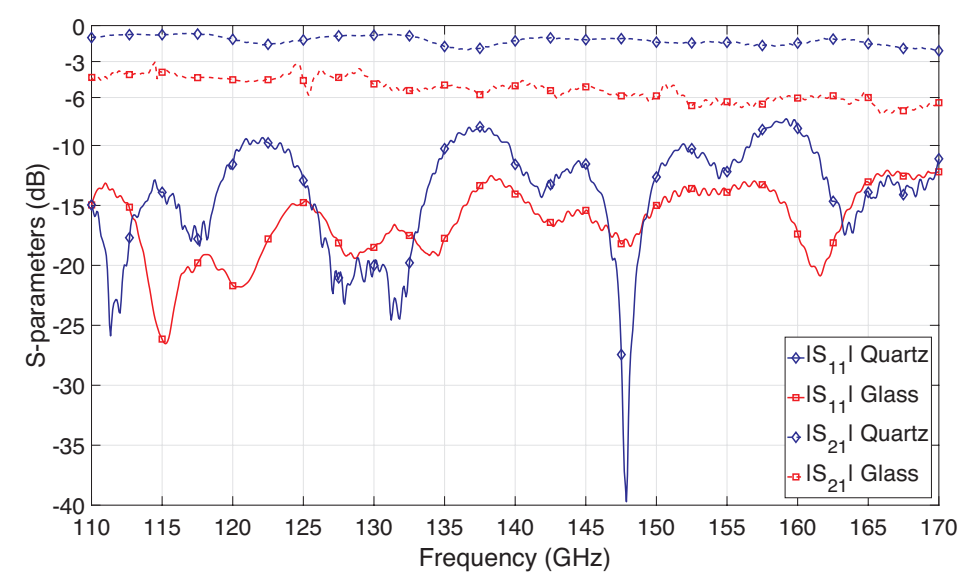

Fig. 3 Measurement results of the fabricated CPWs based on quartz and glass substrates.
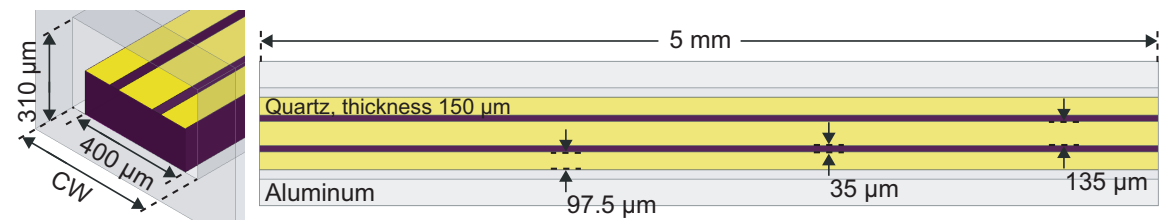

Fig. 4 CPW packaged inside an aluminum cover.

\subsection{Packaged CPW Structure}

When millimeter-wave and submillimeter-wave circuits are packaged into systems, the environment conditions are normally changed from free space to a closed cavity. Fig. 4 shows the situation when a CPW is packaged inside an aluminum cover. In order to achieve a characteristic impedance of $50 \Omega$, the width of the signal trace and the width of the gap are optimized to $135 \mu \mathrm{m}$ and $35 \mu \mathrm{m}$, respectively. Besides, for suppressing parasitic modes inside the packaged cavity, the width of the quartz substrate is reduced to $400 \mu \mathrm{m}$, which results in a width of $97.5 \mu \mathrm{m}$ for the ground traces. The height of the cavity is $310 \mu \mathrm{m}$ and the width is represented by $C W$. When the width of the cavity is the same as the width of the substrate $(C W=400 \mu \mathrm{m})$ the ground traces touch the sidewalls of the aluminum cover providing ground connections to the packaged CPW otherwise the ground traces are floating.

For easier alignment and packaging processes, the width of the cavity, as fabricated by milling, is slightly larger than the width of the diced substrate. Fig. 5 shows the simulation results of the packaged CPW with different $C W$ values. When the cavity is wider than the substrate, due to parasitic modes caused by the floating ground traces of the packaged CPW, the resonances appear periodically. Under this circumstance, silver conductive glue can be used for filling the gaps between the sidewalls of the cavity and the substrate. 


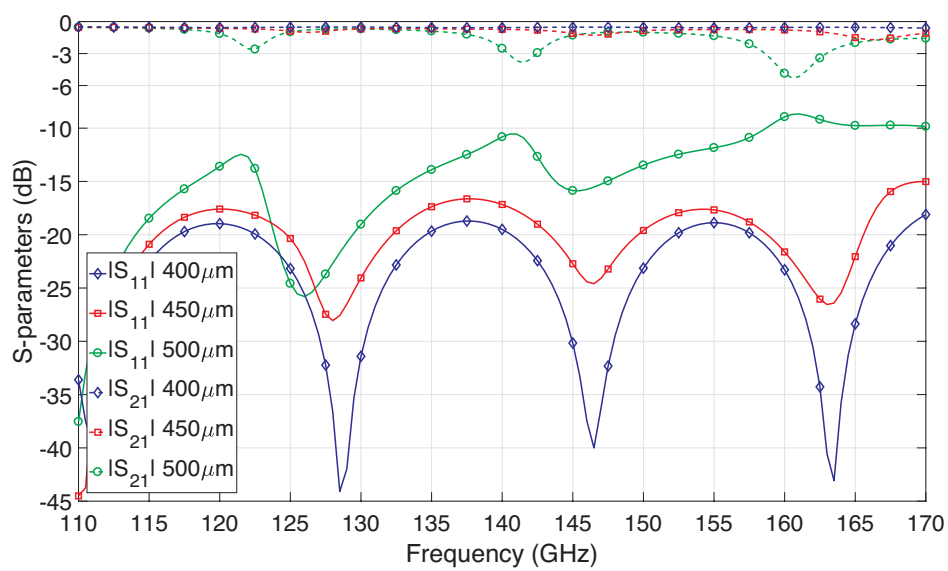

Fig. 5 Simulation results of the packaged CPW with different $C W$ values.

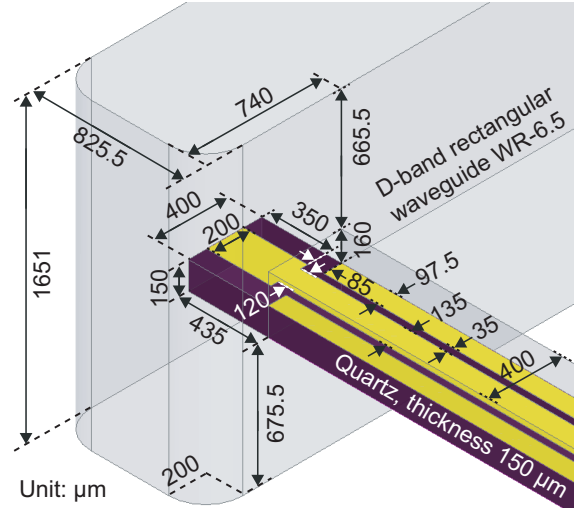

(a)

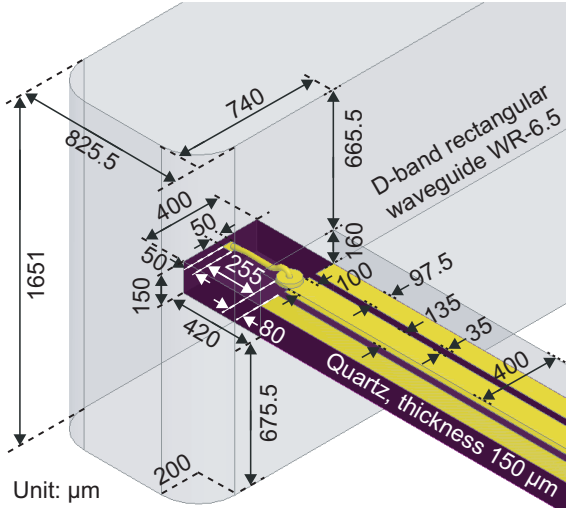

(b)

Fig. 6 Rectangular waveguide-to-CPW transitions at D-band. a E-plane probe. b Wire bonding probe.

\section{Rectangular Waveguide-to-CPW Transitions}

\subsection{E-plane Probe Transition}

Fig. 6a shows the designed rectangular waveguide-to-CPW transition at Dband using E-plane probe. The transition is designed as a reference based on the existing technology where a rectangular patch works as a probe and it is connected to the signal trace of the CPW. For system packaging, the CPW included in the designed transition needs to be connected to other chips.

The dimensions of the packaged CPW and the quartz substrate are the same as those shown in Fig. 4. The width and length of the rectangular patch are $200 \mu \mathrm{m}$ and $350 \mu \mathrm{m}$, respectively. By changing the size of the rectangular patch, the probe can be adjusted to different frequency bands. For matching 
the rectangular patch, the signal trace is extended with a length of $85 \mu \mathrm{m}$ and a width of $120 \mu \mathrm{m}$. The designed E-plane probe is inserted into a WR-6.5 rectangular waveguide through an aperture cut which is located $740 \mu \mathrm{m}$ away from the end of the waveguide. The width and height of a WR-6.5 rectangular waveguide are $1.651 \mathrm{~mm}$ and $0.8255 \mathrm{~mm}$, respectively. Since the packaging structure is fabricated by milling which is carried out using a spinner with a diameter of $400 \mu \mathrm{m}$, the radius of the rounded corners at the end of the rectangular waveguide is $200 \mu \mathrm{m}$. The cavity has the same width as the substrate while it is slightly larger for the fabricated packaging structure due to substrate alignment and fabrication tolerances. Silver conductive glue is used for providing ground connections to the CPW and suppressing parasitic modes in the cavity. Besides, it helps to fix the substrate inside the packaging structure.

\subsection{Wire Bonding Probe Transition}

Though wire bondings are widely used as connections for system integrations between different components and chips, it introduces significant parasitic inductances especially at millimeter-wave and submillimeter-wave frequencies which as a result, limits its usage. Fig. $6 \mathrm{~b}$ shows the proposed transition at Dband in which the wire bonding itself works as a probe guiding electromagnetic waves from the rectangular waveguide to the CPW. For system packaging, the CPW in the proposed transition can be replaced directly by other chips.

The rectangular waveguide and the packaged CPW are the same as those used for the E-plane probe transition in Fig. 6a. In this way, the performances of different transitions can be compared since the aluminum packaging structure used for the E-plane probe is reused for the wire bonding probe. Instead of the rectangular patch, a wire bonding with a diameter of $25 \mu \mathrm{m}$ is used as a probe inserting into the rectangular waveguide. For making a wire bonding between two positions, a conductor ball needs to be soldered and pressed on the substrate at the first position for fixing the wire bonding. Thus the signal trace of the CPW is extended $80 \mu \mathrm{m}$ in length and $100 \mu \mathrm{m}$ in width. At the second position, a square pad with a length of $50 \mu \mathrm{m}$ is located $255 \mu \mathrm{m}$ away from the extended pad and it is reserved for terminating the wire bonding which also helps to control the length of the wire bonding. By adjusting the length as well as the shape, the wire bonding probe can be configured to operate at different frequency bands. Besides, the proposed wire bonding probe can be connected directly to the chip which avoids the use of transmission lines as well as connections between the chip and the probe.

Since controlling the shape of a wire bonding is not as easy as controlling the length, the shape of the wire bonding probe used for the proposed transition shown in Fig. $6 \mathrm{~b}$ is based on the real shape of a fabricated wire bonding with a similar length. Fig. 7a shows the details of the modeled wire bonding probe whose shape is described by six points on a curve. The curve has a fixed length and the modeled wire bonding probe can be configured to different shapes by changing the positions of the points. In order to address the 


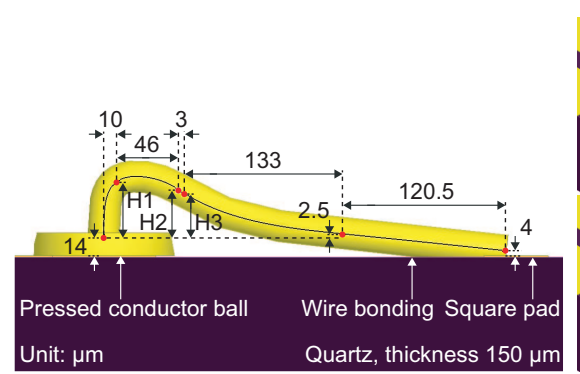

(a)

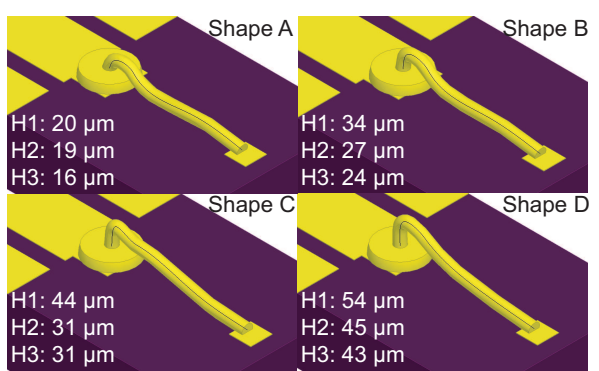

(b)

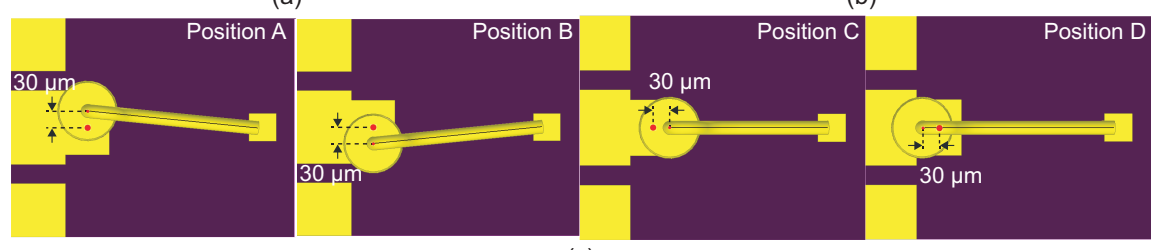

(c)

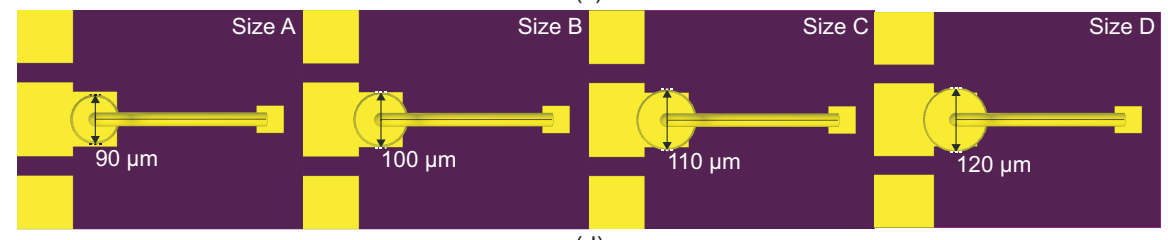

(d)

Fig. 7 a Modeled wire bonding probe. b Different shapes of the wire bonding probe. c Different positions of the wire bonding probe. $\mathbf{d}$ Different diameters of the pressed conductor ball.

performance sensitivity against fabrication tolerances, the proposed transition at D-band using wire bonding probe is simulated with different shapes, positions, and conductor ball diameters. The shape variations are demonstrated in Fig. 7b. By moving the points on the curve vertically to higher positions, the total height of the wire bonding probe increases from $53 \mu \mathrm{m}$ to $83 \mu \mathrm{m}$. When the length of the wire bonding probe is fixed, increasing the height results in a larger occupation area in the vertical plane which moves the wire bonding probe to lower frequencies. In addition, Fig. 7c illustrates the cases when the wire bonding probe has a misalignment of $30 \mu \mathrm{m}$ in different directions. As is shown in Fig. 7d, due to the force variations, the pressed conductor ball might have different diameters which ranges from $90 \mu \mathrm{m}$ to $120 \mu \mathrm{m}$.

The simulation results of the proposed transition at D-band using wire bonding probe with different shapes, positions, and conductor ball diameters are compared in Fig. 8. When the shape of the wire bonding probe is changed by a height variation of approximate $30 \mu \mathrm{m}$, the bandwidth of the proposed transition shifts $7.5 \mathrm{GHz}$ at D-band. For the misaligned positions with a distance of $30 \mu \mathrm{m}$ from the origin, the frequency shift on the simulation results are still acceptable which are partly caused by the length variation of the wire bonding probe. Besides, when the outline of the pressed conductor ball is lo- 
cated within the edges of the extended pad on the signal trace, the effects on the simulation results can be neglected otherwise it might cause mismatch and affect the bandwidth.

\subsection{System Packaging Approaches}

Since both E-plane probe and wire bonding probe can be used for rectangular waveguide-to-CPW transitions at different frequency bands, their system packaging approaches are demonstrated and compared in Fig. 9.

For on-chip approach using E-plane probe, the probe is patterned on the chip substrate which does not require extra connections between the transmission line and the chip. In this case, a low-loss transmission can be achieved but the fabricated chip cannot be tested individually before it packaged with rectangular waveguides which is a risk when several chips are integrated into a system. It becomes difficult to adjust one of the chips or to locate the position of the problem. Besides, this approach is not suitable for packaging the existing chips since these chips do not contain any on-chip E-plane probe when they were fabricated. Though carrier substrate approach using E-plane probe solves the problem of individual testing and it can be used for packaging the existing chips as well, the connections between the transition and the chip are the main concern. Such connections using either wire bondings or vias introduce parasitic inductances and limit the bandwidth of the packaged system especially at high frequencies.

For on-chip approach using wire bonding probe, the square pad for terminating the wire bonding probe is patterned on the chip substrate and it does not require extra transmission lines or connections. Since the square pad is independent from the circuit, the chip can also be tested individually before it is packaged into a system. Carrier substrate approach using wire bonding probe is suitable for packaging the existing chips where the chip on the top is connected to the square pad on the bottom substrate through a wire bonding. Compared with the E-plane probe transition, the wire bonding probe transition is more versatile and compact which supports both on-chip patterning and individual testing at the same time without extra transmission lines and connections between the probe and the chip.

\section{Fabrication and Experimental Results}

\subsection{Downscaled Transitions at U-Band}

In order to validate the concept, a rectangular waveguide-to-CPW transition using wire bonding probe was preliminarily designed at U-band (40-60 GHz) and its design details as well as simulation results were reported in [20]. Fig. 10 shows the fabricated transitions in a back-to-back configuration. The length of the CPW in the middle is $20 \mathrm{~mm}$ and the length of the WR-19 rectangular 


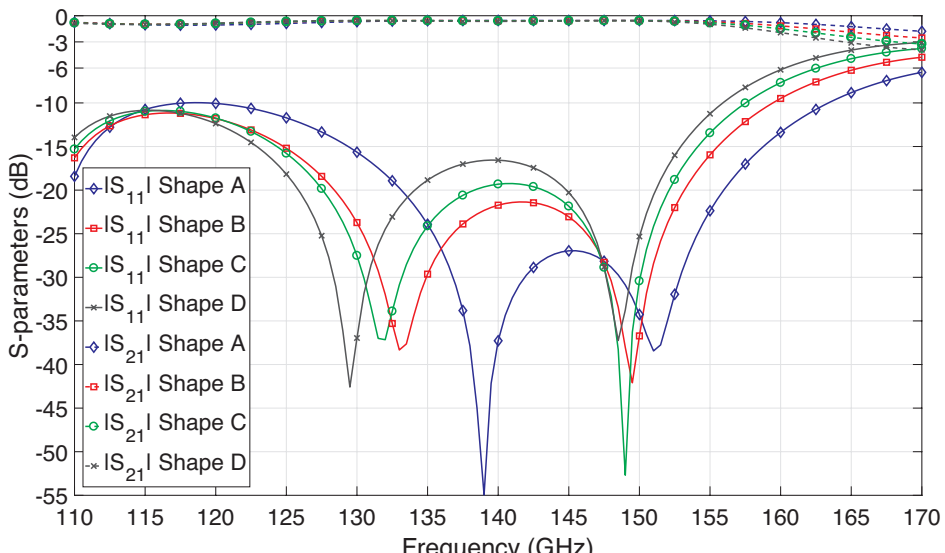

(a)

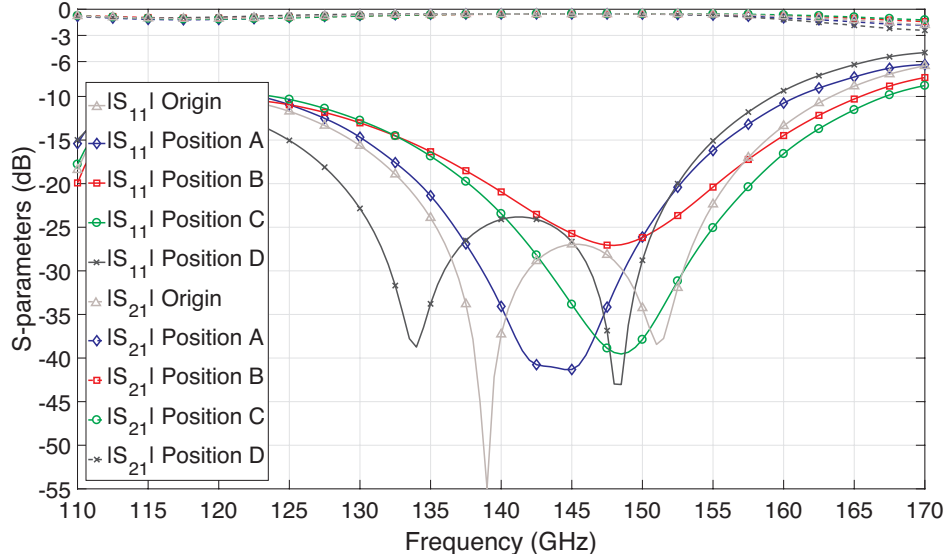

(b)

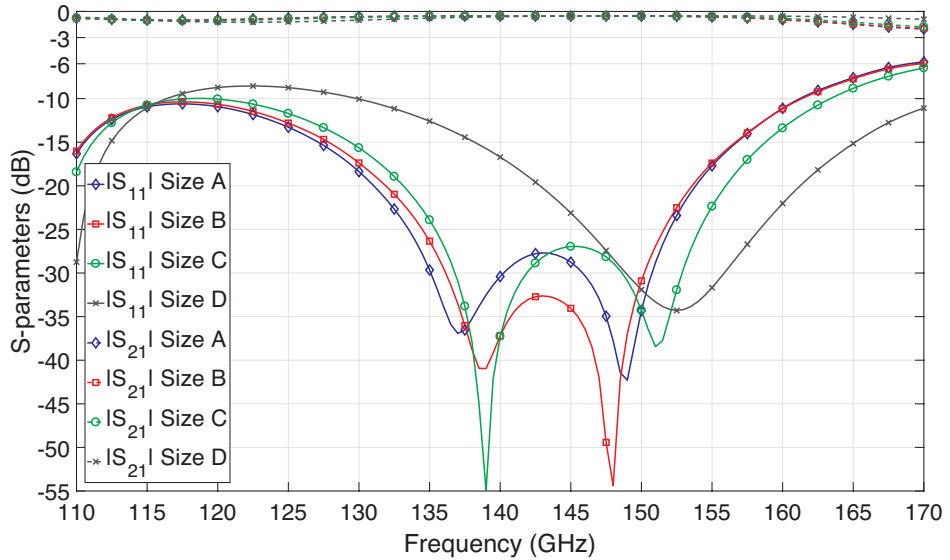

(c)

Fig. 8 Simulation results of the rectangular waveguide-to-CPW transition at D-band. a Different shapes of the wire bonding probe. $\mathbf{b}$ Different positions of the wire bonding probe. c Different diameters of the pressed conductor ball. 

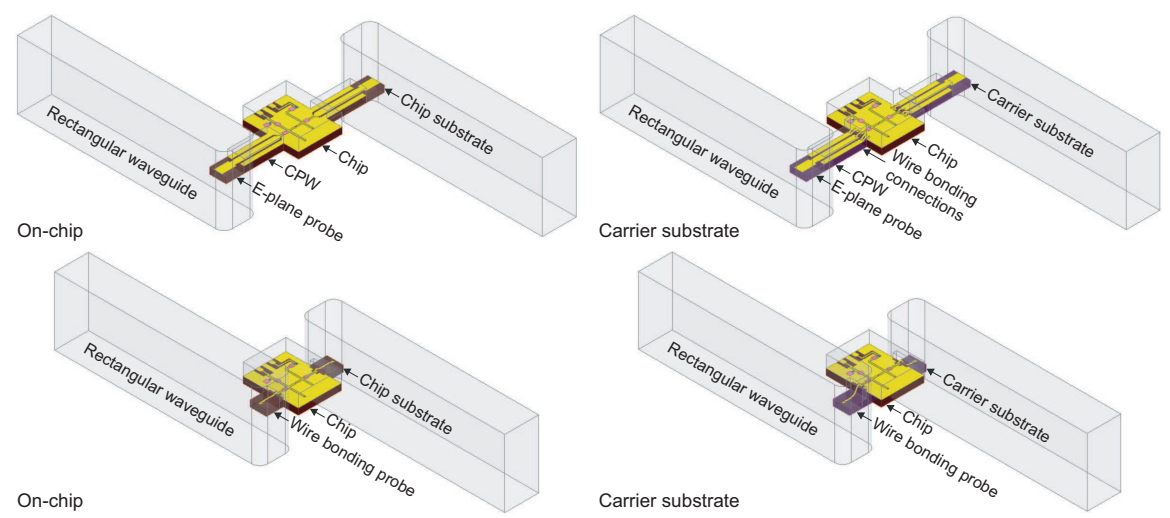

Fig. 9 System packaging approaches using E-plane probe and wire bonding probe.

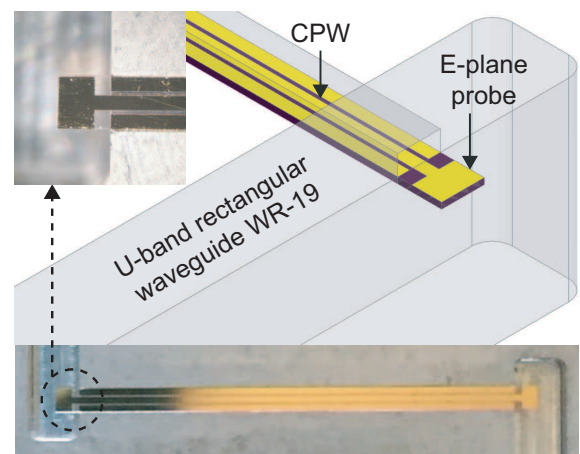

(a)

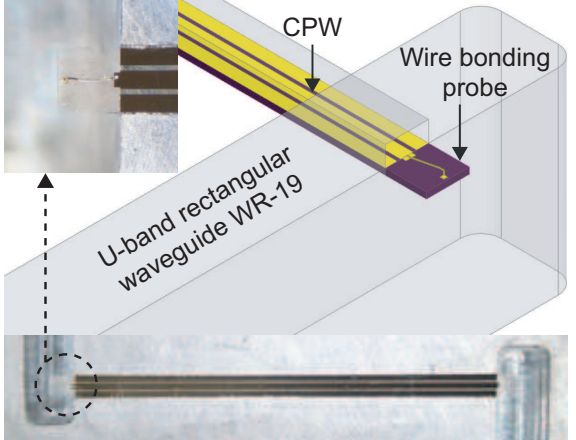

(b)

Fig. 10 Fabricated rectangular waveguide-to-CPW transitions at U-band in a back-to-back configuration. a E-plane probe. b Wire bonding probe.

waveguide is $50 \mathrm{~mm}$.

The fabricated transitions were measured from $40 \mathrm{GHz}$ to $60 \mathrm{GHz}$ and the results are compared in Fig. 11. The transition using wire bonding probe exhibits a bandwidth of $11 \mathrm{GHz}$. The return loss is better than $10 \mathrm{~dB}$ from $49 \mathrm{GHz}$ to $60 \mathrm{GHz}$ with an associated insertion loss of $3 \mathrm{~dB}$. As a reference, a bandwidth of $18 \mathrm{GHz}$ is achieved by the transition using E-plane probe.

\subsection{Fabricated Transitions at D-Band}

The rectangular waveguide-to-CPW transitions shown in Fig. 6 were fabricated in a back-to-back configuration. The packaging structure of the designed transitions at D-band is shown in Fig. 12. It consists of two WR-6.5 rectangular waveguides with a length of $20 \mathrm{~mm}$ and a cavity in the middle with a length of $2 \mathrm{~mm}$. Due to the fabrication and substrate alignment processes, the packaging structure was divided into two parts and they were fabricated using 


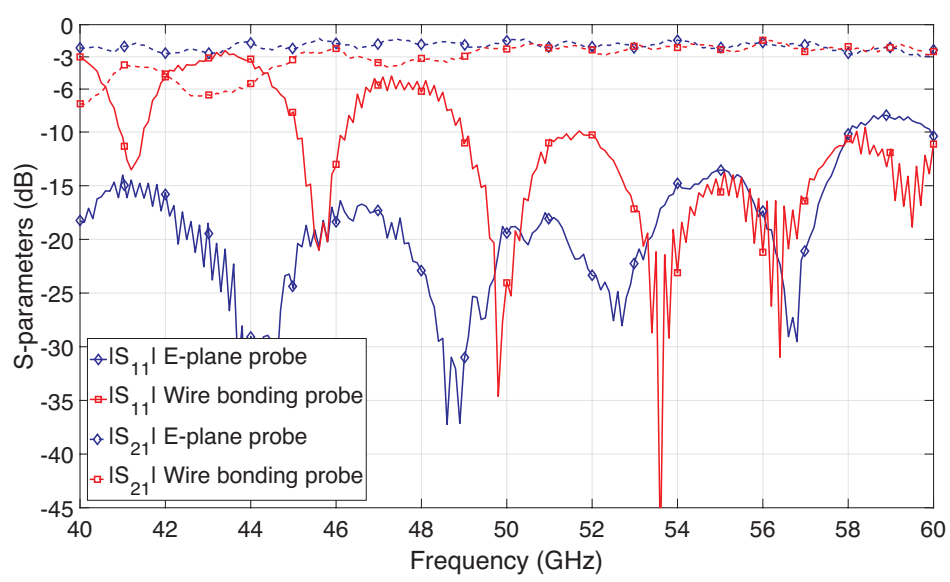

Fig. 11 Measurement results of the fabricated transitions at U-band using E-plane probe and wire bonding probe in a back-to-back configuration.
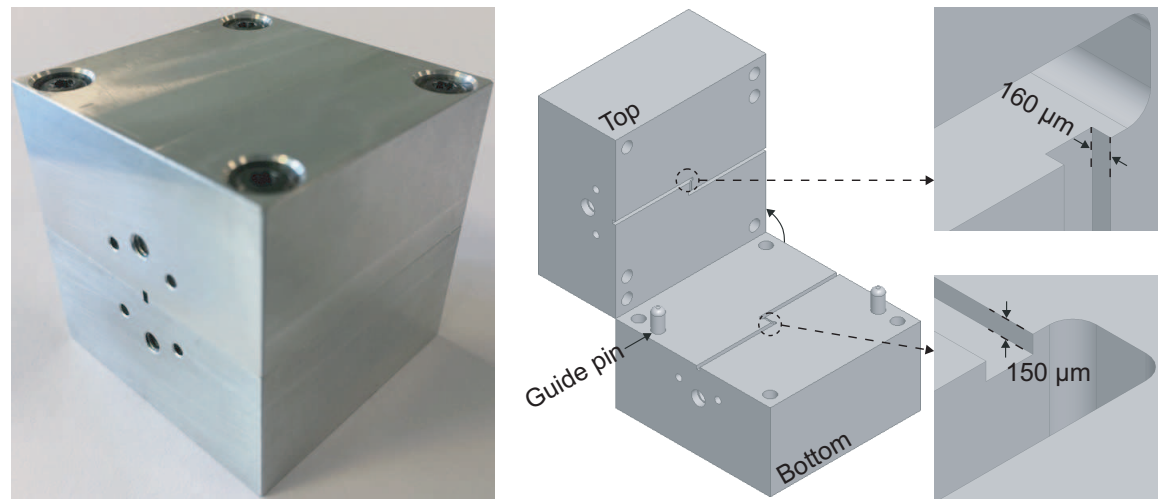

Fig. 12 Fabricated aluminum package of the designed transitions at D-band.

two aluminum blocks by milling. Two guide pins and four screws were used for accurate assembly. The depths of the slots on the top and bottom parts are $160 \mu \mathrm{m}$ and $150 \mu \mathrm{m}$, respectively. The standard WR-6.5 rectangular waveguide flanges without annular recess were applied for the fabricated aluminum package.

The transitions using E-plane probe and wire bonding probe were fabricated at Danchip using a blank quartz substrate with a thickness of $150 \mu \mathrm{m}$. The substrate is $25 \mathrm{~mm}$ in length and width. During the fabrication processes, the quartz substrate was first cleaned by sputtering. Then a titanium layer of $30 \mathrm{~nm}$ and a gold layer of $400 \mathrm{~nm}$ were deposited on the substrate. By using laser ablation, the proposed transitions were patterned on the quartz substrate. For each transition design, several samples were patterned on the substrate and they were diced into individual pieces. Since the quartz substrate is fragile, it was sticked on top of a silicon wafer and they were diced 


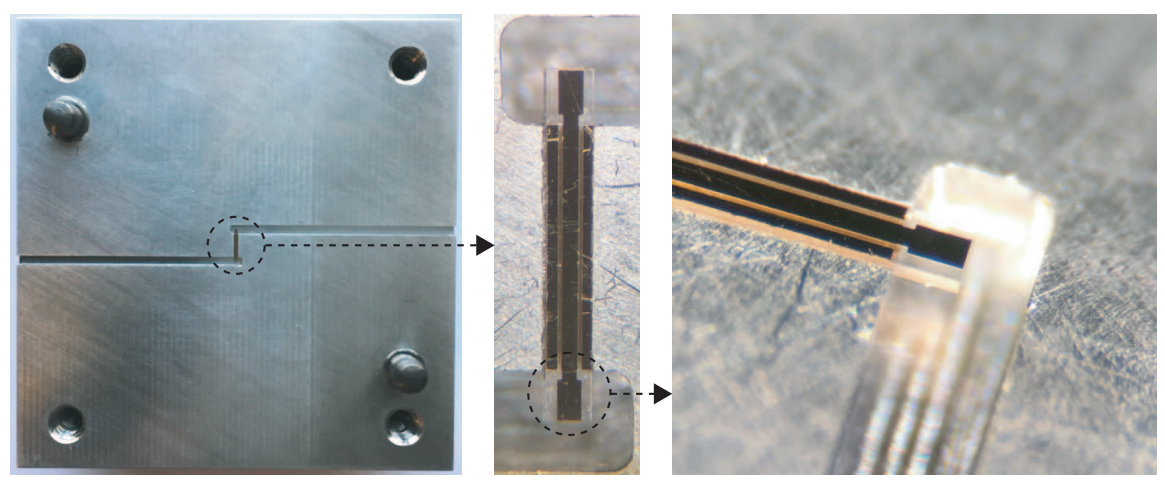

(a)

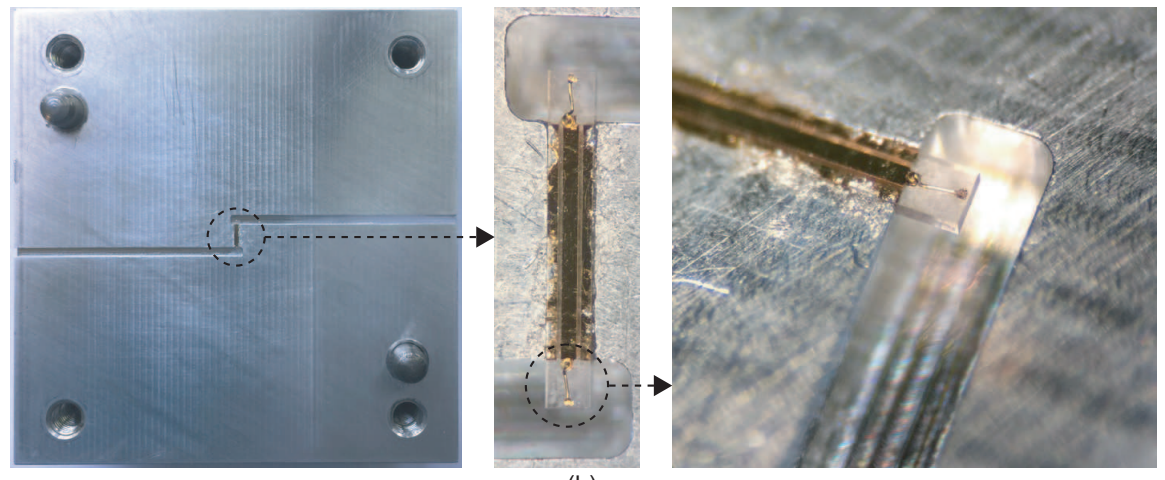

(b)

Fig. 13 Fabricated rectangular waveguide-to-CPW transitions at D-band in a back-to-back configuration. a E-plane probe. b Wire bonding probe.

together in order to overcome the stress during the dicing process. The wire bondings were made at the last step on the diced samples. As is shown in Fig. 13, the fabricated transitions were aligned inside the bottom slot of the aluminum package where the depth of the slot is the same as the thickness of the quartz substrate. The length of the CPW in the middle of the transition is $2 \mathrm{~mm}$. The gaps between the substrate and the sidewalls of the slot were filled with silver conductive glue. Since the same aluminum package was reused for different transitions, it was cleaned by acetone which works as a solvent for dissolving the silver conductive glue in the gaps.

\subsection{Experimental Setup}

The experimental setup for measuring the proposed transitions at D-band is shown in Fig. 14. The measurement system comprises an Anritsu ME7808B vector network analyzer (VNA) and two VDI WR-6.5 waveguide extenders which can measure two-port scattering parameters from $110 \mathrm{GHz}$ to $170 \mathrm{GHz}$ for the device under test (DUT). In order to improve the accuracy, through- 

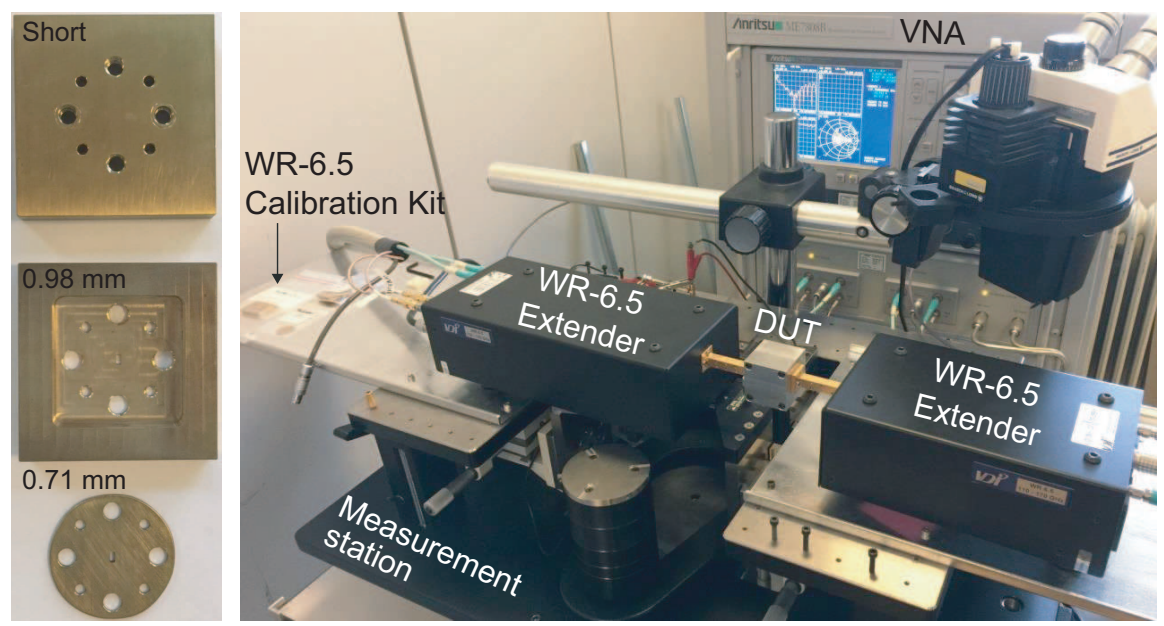

Fig. 14 Experimental setup showing the Anritsu vector network analyzer, VDI WR-6.5 waveguide extenders, and calibration kit.

reflect-line (TRL) calibration is implemented for the measurement and the measured scattering parameters can be calibrated to the input and output of the DUT. The calibration kit consists of a short and two WR-6.5 rectangular waveguides with lengths of $0.98 \mathrm{~mm}$ and $0.71 \mathrm{~mm}$, respectively. When two waveguides cascade together, another WR-6.5 rectangular waveguide with a length of $1.69 \mathrm{~mm}$ can be formed. The material of the calibration kit is brass and it is fabricated by milling with a diameter of $400 \mu \mathrm{m}$.

\subsection{Measurement Results}

Fig. 15 shows the simulation and measurement results of the designed transition at D-band using E-plane probe in a back-to-back configuration. Since the bandwidth refers to the width of the frequency band where the return loss is better than $10 \mathrm{~dB}$, a simulated bandwidth of $60 \mathrm{GHz}$ is shown by the dashed lines from $110 \mathrm{GHz}$ to $170 \mathrm{GHz}$ while the simulated insertion loss is less than $0.7 \mathrm{~dB}$. The solid lines represent the measurement results of the fabricated transition using E-plane probe in a back-to-back configuration. As a reference, it achieves a bandwidth of $57.3 \mathrm{GHz}$ ranging from $112.7 \mathrm{GHz}$ to $170 \mathrm{GHz}$ with an associated insertion loss of $1.6 \mathrm{~dB}$.

The simulation and measurement results of the proposed transition at Dband using wire bonding probe in a back-to-back configuration are shown in Fig. 16 by the dashed and solid lines, respectively. The simulated return loss is better than $10 \mathrm{~dB}$ from $110 \mathrm{GHz}$ to $163.5 \mathrm{GHz}$, which corresponds to a bandwidth of $53.5 \mathrm{GHz}$ while the simulated insertion loss is less than $1.1 \mathrm{~dB}$. The solid lines are compared with the dashed lines and a good agreement has been achieved. The measured bandwidth of the fabricated transition using wire bonding probe in a back-to-back configuration is $56.3 \mathrm{GHz}$ ranging 


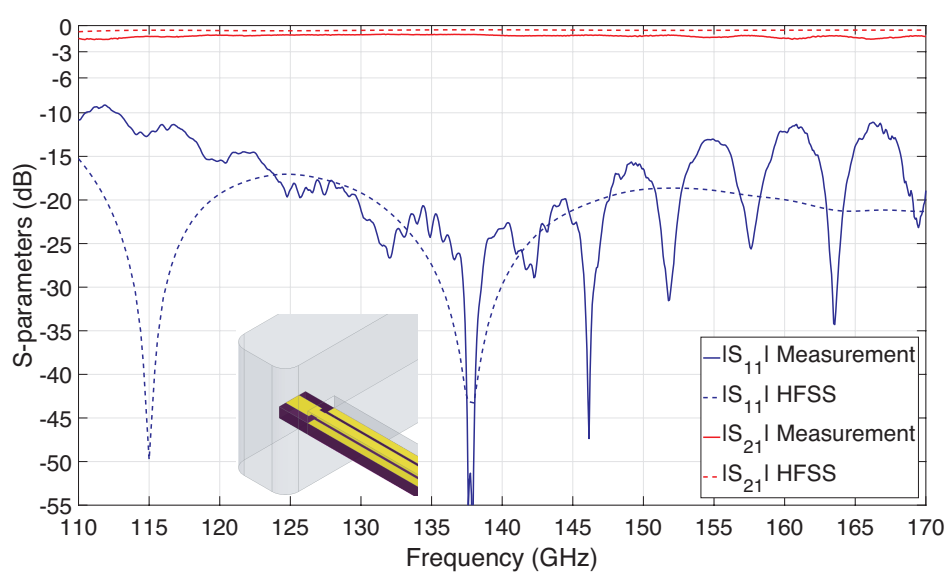

Fig. 15 Simulation and measurement results of the designed transition at D-band using E-plane probe in a back-to-back configuration.

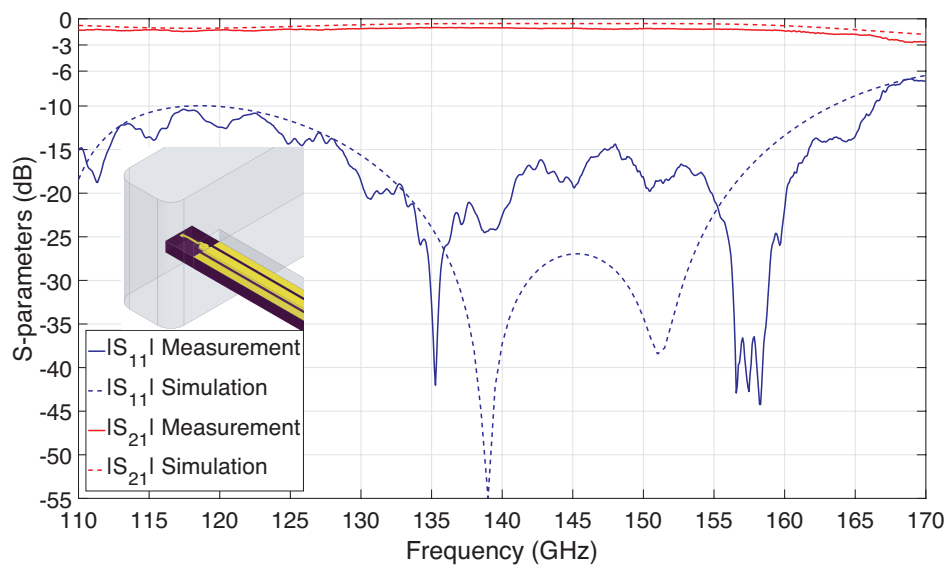

Fig. 16 Simulation and measurement results of the proposed transition at D-band using wire bonding probe in a back-to-back configuration.

from $110 \mathrm{GHz}$ to $166.3 \mathrm{GHz}$ with an associated insertion loss of $2 \mathrm{~dB}$. The differences between the simulation and measurement results are caused by the variation of the substrate properties based on the operating frequency as well as the fabrication and alignment tolerances, especially for the shape of the wire bonding probes and the gaps between the sidewalls of the cavity and the quartz substrate.

Compared with the fabricated transition using E-plane probe, a comparable performance has been achieved at D-band by the fabricated transition using wire bonding probe. The measured bandwidths of the transitions using E-plane probe and wire bonding probe are $57.3 \mathrm{GHz}$ and $56.3 \mathrm{GHz}$, respectively. For both fabricated transitions, the measured insertion losses are less 
than $3 \mathrm{~dB}$ at D-band while they are less than $2 \mathrm{~dB}$ within the frequency band where the return loss is better than $10 \mathrm{~dB}$.

\section{Conclusion}

In this work, a D-band rectangular waveguide-to-CPW transition using wire bonding probe has been presented. A conventional CPW has been designed and fabricated based on both quartz and glass substrates. The fabrication processes including metal deposition, laser ablation, and dicing were also introduced. An insertion loss of $0.254 \mathrm{~dB} / \mathrm{mm}$ has been achieved by the fabricated $\mathrm{CPW}$ based on quartz substrate. The concept of designing a CPW packaged inside an aluminum cover was explained and the potential risk of parasitic modes was discussed. The designs of rectangular waveguide-to-CPW transitions using E-plane probe and wire bonding probe were described in detail. The system packaging approaches using E-plane probe and wire bonding probe were compared. The proposed rectangular waveguide-to-CPW transitions have been fabricated and measured in a back-to-back configuration in which the transition using E-plane probe is used as a reference. The assembly structure was illustrated and the aluminum package was reused for different transitions. Besides, the experimental setup for measuring two-port scattering parameters at D-band and the corresponding WR-6.5 waveguide calibration kit were described. A good agreement between simulation and measurement has been achieved. The fabricated rectangular waveguide-to-CPW transition at D-band using wire bonding probe in a back-to-back configuration can provide a bandwidth of $56.3 \mathrm{GHz}$ ranging from $110 \mathrm{GHz}$ to $166.3 \mathrm{GHz}$ in which the return loss is better than $10 \mathrm{~dB}$ and the insertion loss is less than $2 \mathrm{~dB}$. Compared with the fabricated transition using E-plane probe, an equivalent bandwidth has been achieved at D-band while the proposed transition using wire bonding probe is more versatile and compact for system packaging.

Acknowledgements The authors would like to thank M. Nielsen, J. Lauridsen, and J. N Mortensen at Technical University of Denmark for machining the packaging structures.

\section{References}

1. T. Tajima, H. J. Song, and M. Yaita, "Design and analysis of LTCC-integrated planar microstrip-to-waveguide transition at $300 \mathrm{GHz}, "$ IEEE Trans. Microw. Theory Techn. vol. 64, no. 1, pp. 106-114, Jan. 2016.

2. B. Zhang and H. Zirath, "Metallic 3-D printed rectangular waveguides for millimeterwave applications," IEEE Trans. Compon. Packag. Manuf. Technol., vol. 6, no. 5, pp. 796-804, May. 2016.

3. J. M. Oliver, J. M. Rollin, K. Vanhille, and S. Raman, "A W-band micromachined 3-D cavity-backed patch antenna array with integrated diode detector," IEEE Trans. Microw. Theory Techn., vol. 60, no. 2, pp. 284-292, Feb. 2012.

4. M. Moallem, J. East, and K. Sarabandi, "A broadband, micromachined rectangular waveguide to cavity-backed coplanar waveguide transition using impedance-taper technique," IEEE Trans. THz Sci. Technol., vol. 4, no. 1, pp. 49-55, Jan. 2014. 
5. N. Vahabisani and M. Daneshmand, "Monolithic wafer-level rectangular waveguide and its transition to coplanar waveguide line using a simplified 3-D fabrication process," IEEE Trans. Compon. Packag. Manuf. Technol., vol. 4, no. 1, pp. 168-176, Jan. 2014.

6. M. Vahidpour and K. Sarabandi, "2.5D micromachined $240 \mathrm{GHz}$ cavity-backed coplanar waveguide to rectangular waveguide transition," IEEE Trans. THz Sci. Technol., vol. 2, no. 3, pp. 315-322, May. 2012.

7. T. Merkle, R. Götzen, J. Y. Choi, and S. Koch, "Polymer multichip module process using 3-D printing technologies for D-band applications," IEEE Trans. Microw. Theory Techn., vol. 63, no. 2, pp. 481-493, Feb. 2015.

8. T. Tajima, H. J. Song, H. Matsuzaki, and M. Yaita, "LTCC-integrated H-plane bends for THz antenna-in-package solution," IEEE Microw. Compon. Lett., vol. 27, no. 5, pp. 440442, May. 2017.

9. H. J. Song, H. Matsuzaki, and M. Yaita, "Sub-millimeter and terahertz-wave packaging for large chip-width MMICs," IEEE Microw. Compon. Lett., vol. 26, no. 6, pp. 422-424, June. 2016

10. M. Varonen, L. Samoska, A. Fung, S. Padmanabhan, P. Kangaslahti, R. Lai, S. Sarkozy, M. Soria, H. Owen, T. Reck, G. Chattopadhyay, P. V. Larkoski, and T. Gaier, "A WR4 amplifier module chain with an $87 \mathrm{~K}$ noise temperature at $228 \mathrm{GHz}$," IEEE Microw. Compon. Lett., vol. 25, no. 1, pp. 58-60, Jan. 2015.

11. M. Kärkkäinen, M. Kantanen, S. C. Bert, M. Varonen, R. Weber, A. Leuther, M. S. Eggebert, A. Alanne, P. Jukkala, T. Närhi, and K. A. I. Halonen, "MHEMT G-band low-noise amplifiers," IEEE Trans. THz Sci. Technol., vol. 4, no. 4, pp. 459-468, July. 2014.

12. V. Hurm, R. Weber, A. Tessmann, H. Massler, A. Leuther, M. Kuri, M. Riessle, H. P. Stulz, M. Zink, M. Schlechtweg, O. Ambacher, and T. Närhi, "A 243 GHz LNA module based on mHEMT MMICs with integrated waveguide transitions," IEEE $M i$ crow. Compon. Lett., vol. 23, no. 9, pp. 486-488, Sept. 2013.

13. J. Stenarson, T. N. T. Do, H. Zhao, P. J. Sobis, A. Y. Tang, K. Yhland, and J. Stake, "Sensitivity analysis of TRL calibration in waveguide integrated membrane circuits," IEEE Trans. THz Sci. Technol., vol. 3, no. 5, pp. 558-565, Sept. 2013.

14. E. S. Li, G. X. Tong, and D. C. Niu, "Full W-band waveguide-to-microstrip transition with new E-plane probe," IEEE Microw. Compon. Lett., vol. 23, no. 1, pp. 4-6, Jan. 2013.

15. L. A. Samoska, "An overview of solid-state integrated circuit amplifiers in the submillimeter-wave and THz regime," IEEE Trans. THz Sci. Technol., vol. 1, no. 1, pp. 9-24, Sept. 2011.

16. O. Donadio, K. Elgaid, and R. Appleby, "Waveguide-to-microstrip transition at G-band using elevated E-plane probe," Electron. Lett., vol. 47, no. 2, pp. 115-116, Jan. 2011.

17. Y. Lee, J. P. Becker, J. R. East, and L. P. B. Katehi, "Fully micromachined finite-ground coplanar line-to-waveguide transitions for W-band applications," IEEE Trans. Microw. Theory Techn., vol. 52, no. 3, pp. 1001-1007, Mar. 2004.

18. S. Jameson and E. Socher, "A wide-band CMOS to waveguide transition at mmwave frequencies with wire-bonds," IEEE Trans. Microw. Theory Techn., vol. 63, no. 9 , pp. 2741-2750, Sept. 2015.

19. K. Eriksson, P. J. Sobis, S. E. Gunnarsson, J. Hanning, and H. Zirath, "InP DHBT amplifier modules operating between 150-300 GHz using membrane technology," IEEE Trans. Microw. Theory Techn., vol. 63, no. 2, pp. 433-440, Feb. 2015.

20. Y. Dong, T. K. Johansen, V. Zhurbenko, and P. J. Hanberg, "Rectangular waveguideto-coplanar waveguide transitions at U-band using E-plane probe and wire bonding," in proc. 46th Eur. Microw. Conf. (EuMC), Oct. 2016, pp. 5-8.

21. M. Tsuji, H. Shigesawa, and A. A. Oliner, "New interesting leakage behavior on coplanar waveguides of finite and infinite widths," IEEE Trans. Microw. Theory Techn., vol. .39, no. 12 , pp. 2130-2137, Dec. 1991.

22. F. Schnieder, T. Tischler, and W. Heinrich, "Modeling dispersion and radiation characteristics of conductor-backed CPW with finite ground width," IEEE Trans. Microw. Theory Techn., vol. .51, no. 1, pp. 137-143, Jan. 2003.

23. A. Sain and K. L. Melde, "Impact of ground via placement in grounded coplanar waveguide interconnects," IEEE Trans. Compon. Packag. Manuf. Technol., vol. 6, no. 1, pp. 136-144, Jan. 2016. 